\title{
Enhancement of nuclear Schiff moments and time reversal violation in atoms due to soft nuclear octupole vibrations
}

\author{
V.V. Flambaum ${ }^{1}$ and V.G. Zelevinsky ${ }^{2}$ \\ ${ }^{1}$ School of Physics, University of New South Wales, Sydney 2052, Australia and \\ Institute for Nuclear Theory, University of Washington, Seattle, WA 98195-1550, USA \\ ${ }^{2}$ National Superconducting Cyclotron Laboratory and \\ Department of Physics and Astronomy, Michigan State University, East Lansing, MI \\ 48824-1321, USA
}

(October 25, 2018)

\begin{abstract}
Parity and time invariance violating $(\mathcal{P}, \mathcal{T}$-odd $)$ nuclear forces produce $\mathcal{P}, \mathcal{T}$-odd nuclear moments, for example, the nuclear Schiff moment. In turn, this moment can induce electric dipole moments (EDMs) in atoms. We estimate the contribution to the Schiff moment from the soft collective octupole vibrations existing in many heavy nuclei. Two possible mechanisms are considered, mixing of the ground state of an odd- $A$ nucleus with the octupole phonon state, and $\mathcal{P}, \mathcal{T}$-odd admixture to the single-particle wave function of the valence nucleon. We found practically the same contribution to the Schiff moment as that of the static octupole deformation calculated earlier. This confirms a suggestion that the soft octupole vibrations can replace the (controversial) static octupoles in development of the collective Schiff moments. The values of atomic EDM predicted for ${ }^{223,225} \mathrm{Ra}$ and ${ }^{223} \mathrm{Rn}$ are enhanced by factors up to $10^{3}$ compared to experimentally studied spherical nuclei ${ }^{199} \mathrm{Hg}$ and ${ }^{129} \mathrm{Xe}$.
\end{abstract}

PACS: 32.80.Ys,21.10.Ky,24.80.+y 


\section{INTRODUCTION}

A discovery of the static electric dipole moment (EDM) of an elementary particle, atom or atomic nucleus would reveal [1] the simultaneous violation of the invariance with respect to spatial inversion $(\mathcal{P})$ and time reversal $(\mathcal{T})$. The best limit on nucleon-nucleon interactions violating parity and time invariance $(\mathcal{P}, \mathcal{T}$-odd forces $)$, as well as quark-quark $\mathcal{P}, \mathcal{T}$-odd interactions, has been obtained from the measurement of the atomic EDM in ${ }^{199} \mathrm{Hg}$ [2] calculated in Ref. [3]. According to the Schiff theorem [1, 1,5], the nuclear EDM is screened by atomic electrons. The EDM of an atom with closed electron subshells is induced by the nuclear Schiff moment [6] that is defined as a mean square radius of the dipole charge distribution with the contribution of the center-of-charge subtracted,

$$
\mathbf{S}=\frac{e}{10}\left[\left\langle r^{2} \mathbf{r}\right\rangle-\frac{5}{3 Z}\left\langle r^{2}\right\rangle\langle\mathbf{r}\rangle\right]
$$

Here $\left\langle r^{n}\right\rangle \equiv \int \rho(\mathbf{r}) r^{n} d^{3} r$ are the moments of the nuclear charge density operator $\rho$. The expectation values of the $\mathcal{P}$-odd vector operators $\left\langle\mathbf{r} r^{2}\right\rangle$ and $\langle\mathbf{r}\rangle$ do not vanish due to the $\mathcal{P}, \mathcal{T}$-odd contribution to the charge density while $\left\langle r^{2}\right\rangle / Z=r_{\mathrm{ch}}^{2}$ is the nuclear mean square charge radius. As a consequence of the rotational invariance, the existence of the non-zero Schiff moment requires a non-zero nuclear spin $\mathbf{I}$,

$$
\mathbf{S}=S \frac{\mathbf{I}}{I}
$$

In a search for nuclear structure mechanisms of the enhancement, it was suggested in Ref. [7] that some actinide nuclei have a level of opposite parity and the same spin close to the ground state, and this may enhance the level mixing and the resulting nuclear EDM. In Ref. [6] a similar suggestion was put forward for the enhancement of the Schiff moment $\mathbf{S}$. It was noticed in Ref. [8] that, in contrast to $\mathcal{P}$-odd $\mathcal{T}$-even forces, the parity doublets present for a static pear-shaped deformation can be directly mixed by $\mathcal{P}, \mathcal{T}$-odd forces. This mixing might be enhanced because of close intrinsic structure within the doublet and relatively small energy splitting. As shown in Refs. [9, 10, nuclei with static octupole deformation may have enhanced collective Schiff moments up to 1000 times larger than the Schiff moments of spherical nuclei. An additional enhancement factor appears in this case due to the large static intrinsic Schiff moment in the body-fixed frame which is proportional to the collective octupole moment.

It was suggested in Ref. [11] that the soft octupole vibrations observed in some regions of the nuclear chart may produce an enhancement similar to that due to static octupole deformation. This would make heavy atoms containing such nuclei with large collective Schiff moments attractive for future experiments in search for $\mathcal{P}, \mathcal{T}$-violation; experiments of this type are currently under progress or in preparation in several laboratories. Below we perform the estimate of the Schiff moment generated in nuclei with the soft octupole mode and show that the result is nearly the same as in the case of the static octupole deformation. We consider two simultaneously acting mechanisms, mixing of the ground state of the odd- $A$ nucleus with the one-phonon octupole state ("core excitation") and mixing of the single-particle wave function of the valence unpaired nucleon with levels of opposite parity through the dynamical octupole deformation ("particle excitation"). Our analytical estimates are based on well known general properties of nuclear structure rather than on specific assumptions concerning the model parameters. 


\section{COLLECTIVE NUCLEAR SCHIFF MOMENT}

Consider a nucleus with two levels, ground state $\mid$ g.s. $\rangle$ and excited state $|x\rangle$, of opposite parity and close energies, $E_{\text {g.s. }}$ and $E_{x}$, respectively. Let $W$ be a $\mathcal{P}, \mathcal{T}$-odd interaction capable of mixing these unperturbed states. Assuming that the mixing matrix elements of $\mathbf{S}$ and $W$

are real, we can write down the Schiff moment emerging in the actual mixed ground state as

$$
\mathbf{S}=2 \frac{\langle\text { g.s. }|W| x\rangle\langle x|\mathbf{S}| \text { g.s. }\rangle}{E_{\text {g.s. }}-E_{x}} .
$$

However, as it was explained in Ref. [6], in the case of mixing of closely lying single-particle states one should not expect a large enhancement. For example, in a simple approximate model, where the strong nuclear potential is proportional to nuclear density and the spinorbit interaction is neglected, the matrix element $\langle$ g.s. $|W| x\rangle$ contains the single-particle momentum operator and is proportional to $\left(E_{\text {g.s. }}-E_{x}\right)$ so that the small energy denominator cancels out. As mentioned above, the collective Schiff moments in nuclei with static octupole deformations may be by 2-3 orders of magnitude stronger than single-particle moments in spherical nuclei.

The mechanism generating the collective Schiff moment is the following [9, 10]. In the "frozen" body-fixed frame the intrinsic collective Schiff moment $S_{\text {intr }}$ can exist without any $\mathcal{P}, \mathcal{T}$-violation. However, in the space-fixed laboratory frame the nucleus has a certain angular momentum rather than orientation, and this makes the expectation value of the Schiff moment vanish in the case of no $\mathcal{P}, \mathcal{T}$-violation. Indeed, the intrinsic Schiff moment is directed along the nuclear axis, $\mathbf{S}_{\text {intr }}=S_{\text {intr }} \mathbf{e}$, and in the laboratory frame the only possible correlation $\langle\mathbf{e}\rangle \propto \mathbf{I}$ violates parity and time reversal invariance. The $\mathcal{P}, \mathcal{T}$-odd nuclear forces mix rotational states $|I, \pm\rangle$ of the same spin and opposite parity and create an average orientation of the nuclear axis e along the nuclear spin $\mathbf{I}$,

$$
\left\langle e_{z}\right\rangle=2 \alpha \frac{K M}{I(I+1)},
$$

where

$$
\alpha=\frac{\langle I-|W| I+\rangle}{E_{+}-E_{-}}
$$

is the mixing amplitude of the states of opposite parity, $K=|\mathbf{I} \cdot \mathbf{e}|$ is the absolute value of the projection of the nuclear spin $\mathbf{I}$ on the nuclear axis, and $M$ is the spin projection onto the laboratory quantization axis. The observable Schiff moment in the laboratory frame is then related to the intrinsic moment as

$$
S_{z}=S_{\mathrm{intr}}\left\langle e_{z}\right\rangle=S_{\mathrm{intr}} \frac{2 \alpha K M}{I(I+1)} .
$$

To estimate the intrinsic Schiff moment, we use the standard description of the surface of an axially symmetric deformed nucleus in the body-fixed frame in terms of the multipole deformation parameters $\beta_{l}$, 


$$
R(\theta)=R\left[1+\sum_{l=1} \beta_{l} Y_{l 0}(\theta)\right]
$$

In order to keep the center-of-mass at the origin we have to fix $\beta_{1}$ [20]:

$$
\beta_{1}=-3 \sqrt{\frac{3}{4 \pi}} \sum_{l=2} \frac{(l+1) \beta_{l} \beta_{l+1}}{\sqrt{(2 l+1)(2 l+3)}} .
$$

We assume that the center of the charge distribution coincides with the center-of-mass, so that the electric dipole moment vanishes, $e\langle\mathbf{r}\rangle=0$, and hence there is no screening contribution to the Schiff moment (no second term in eq. (1)). We also assume a constant density for $r<R(\theta)$. The intrinsic Schiff moment $S_{\text {intr }}$ is then [9, 10]

$$
S_{\mathrm{intr}}=e Z R^{3} \frac{3}{20 \pi} \sum_{l=2} \frac{(l+1) \beta_{l} \beta_{l+1}}{\sqrt{(2 l+1)(2 l+3)}} \approx \frac{9}{20 \pi \sqrt{35}} e Z R^{3} \beta_{2} \beta_{3}
$$

where the major contribution comes from the product $\beta_{2} \beta_{3}$ of the static quadrupole, $\beta_{2}$, and octupole, $\beta_{3}$, deformation parameters. For $\beta_{2} \sim \beta_{3} \sim 0.1$ and $Z=88$ (Ra) we obtain $S_{\text {intr }} \sim 10 \mathrm{efm}^{3}$. The estimate of the Schiff moment in the laboratory frame $[K=M=I$ in Eq. (6)] gives [10]

$$
S=S_{\text {intr }} \frac{2 \alpha I}{I+1} \sim 0.01 \frac{I}{I+1} e \beta_{2} \beta_{3}^{2} Z A^{2 / 3} \frac{\eta G}{m r_{0}\left(E_{+}-E_{-}\right)},
$$

where $\eta G$ is the strength constant of the $\mathcal{P}, \mathcal{T}$-odd nuclear potential, traditionally introduced with the aid of the Fermi weak interaction constant $G, R=r_{0} A^{1 / 3}, A$ is the nuclear mass number, and $r_{0} \approx 1.2 \mathrm{fm}$ is the internucleon distance. For the isotope ${ }^{225} \mathrm{Ra}$, where $E_{+}-$ $E_{-}=55 \mathrm{keV}$ and $I=1 / 2$, this analytical estimate gives the Schiff moment $S \sim 500$ in units $10^{-8}$ ne $\mathrm{fm}^{3}$; for ${ }^{223} \mathrm{Ra}\left(E_{+}-E_{-}=50 \mathrm{keV}, I=3 / 2\right) S \sim 1000$. The numerical calculation [10] gives $S=300$ for ${ }^{225} \mathrm{Ra}, 400$ for ${ }^{223} \mathrm{Ra}$ and 1000 for ${ }^{223} \mathrm{Rn}$. To indicate the accuracy of the calculation we should note that the difference between the values obtained for the Woods-Saxon potential (presented above) and Nilsson potential is within a factor of 2 [13.

The values we obtained are several hundred times larger than the Schiff moment of a spherical nucleus like $\mathrm{Hg}(S=-1.4)$. An additional enhancement of the atomic EDM appears due to the greater nuclear charge in Ra and $\mathrm{Rn}$ and close atomic states of opposite parity [15,16]. Accurate atomic calculations of the EDM for atoms of $\mathrm{Hg}, \mathrm{Xe}, \mathrm{Ra}, \mathrm{Rn}$, and $\mathrm{Pu}$ have been performed in Ref. [14].

$\mathcal{T}$ and $\mathcal{P}$-odd nuclear forces can also produce enhanced collective magnetic quadrupole [17 and octupole 18 moments. Although these moments cannot induce the EDM in the ground state of the closed-shell atoms like $\mathrm{Xe}, \mathrm{Hg}, \mathrm{Rn}$ and $\mathrm{Ra}$, they can contribute to the EDM of metastable excited states or of the ground states in open-shell atoms.

Note that the Schiff moment $S$ in Eq. (10) is proportional to the squared octupole deformation parameter $\beta_{3}^{2}$. According to [11, in nuclei with a soft octupole vibration mode the dynamical octupole deformation is of the order of $\left\langle\beta_{3}^{2}\right\rangle \sim(0.1)^{2}$, i.e. the same as the static octupole deformation in pear-shaped nuclei. This means that a number of heavy nuclei can have large collective Schiff moments of dynamical origin. A calculation of these moments is the main subject of the present paper. 


\section{SCHIFF MOMENT PRODUCED BY SOFT OCTUPOLE VIBRATIONS}

A fully microscopic calculation of the Schiff moment in odd- $A$ actinides, even with the aid of simplifying models, such as the particle-plus-rotor model [19, is very complicated, requires also the solution of the random phase approximation for the soft octupole mode in adjacent even-even isotopes and contains many uncertainties in the choice of the parameters. For our purpose of estimating the magnitude of the effect we use below simple analytical arguments.

The intrinsic nuclear octupole moment in the body-fixed frame is given by [20]

$$
O_{\text {intr }}=e \int \rho r^{3} Y_{30} d^{3} r \approx \frac{3}{4 \pi} e Z R^{3} \beta_{3}
$$

The intrinsic Schiff moment $S_{\text {intr }}$, Eq. (9), is then

$$
S_{\text {intr }}=\frac{3}{5 \sqrt{35}} O_{\text {intr }} \beta_{2}
$$

This relation allows us to approximately express the matrix elements of the Schiff moment operator in odd- $A$ nuclei with static axially symmetric quadrupole deformation $\beta_{2}$ in terms

of the matrix elements of the octupole operator which can be extracted from the observed probabilities of the octupole transitions in an even-even neighbor. In a similar way, as a characteristic of the effective dynamic deformation parameter $\beta_{3}$ in a soft nucleus, it is convenient to use the r.m.s. value extracted from the reduced octupole transition probability $\mathrm{B}(\mathrm{E} 3)$ 21],

$$
\mathrm{B}(\mathrm{E} 3)_{0 \rightarrow 3}=\left|\left\langle 1\left|O_{\text {intr }}\right| 0\right\rangle\right|^{2} \approx\left(\frac{3}{4 \pi} e Z R^{3}\right)^{2}\left\langle\beta_{3}^{\text {rms }}\right\rangle^{2}
$$

Here $|1\rangle$ is a low-lying collective ("one-phonon") octupole excited state.

To find the $\mathcal{P}, \mathcal{T}$-odd Schiff moment (3) we need to know the matrix elements of the $\mathcal{P}, \mathcal{T}$-odd nucleon-nucleon interaction. To the first order in the velocities $p / m$, the $\mathcal{P}, \mathcal{T}$-odd interaction can be presented as [6]

$$
\hat{W}_{a b}=\frac{G}{\sqrt{2}} \frac{1}{2 m}\left(\left(\eta_{a b} \boldsymbol{\sigma}_{a}-\eta_{b a} \boldsymbol{\sigma}_{b}\right) \cdot \nabla_{a} \delta\left(\mathbf{r}_{a}-\mathbf{r}_{b}\right)+\eta_{a b}^{\prime}\left[\boldsymbol{\sigma}_{a} \times \boldsymbol{\sigma}_{b}\right] \cdot\left\{\left(\mathbf{p}_{a}-\mathbf{p}_{b}\right), \delta\left(\mathbf{r}_{a}-\mathbf{r}_{b}\right)\right\}\right),
$$

where $\{$,$\} is an anticommutator, G$ is the Fermi constant of the weak interaction, $m$ is the nucleon mass, and $\boldsymbol{\sigma}_{a, b}, \mathbf{r}_{a, b}$, and $\mathbf{p}_{a, b}$ are the spins, coordinates, and momenta, respectively, of the interacting nucleons $a$ and $b$. The dimensionless constants $\eta_{a b}$ and $\eta_{a b}^{\prime}$ characterize the strength of the $\mathcal{P}, \mathcal{T}$-odd nuclear forces; experiments on measurement of the EDMs are aimed at extracting the values of these constants.

In the context of our problem one can envisage two efficient mechanisms generating the observable $\mathcal{P}, \mathcal{T}$-odd effects. Firstly, the octupole component of the $\mathcal{P}, \mathcal{T}$-odd field produced by the valence nucleon can excite the soft octupole excitation in the core. For brevity we will call this mechanism "core excitation". The second mechanism suggested in Ref. [11, "particle excitation", results from the dynamic octupole deformation of the nuclear mean 
field that mixes the opposite parity orbitals of the valence nucleon and leads to a nonvanishing expectation value of the $\mathcal{P}, \mathcal{T}$-odd interaction in the fixed-body frame. As we will show, the value of the Schiff moment induced by the second mechanism is practically identical to that obtained earlier in Refs. [9, 10] for the case of static octupole deformation. We start with the estimate for the first mechanism.

\section{A. Core excitation}

Let us consider the interaction $W_{a b}$ between the valence nucleon $b$ and the even-even core containing paired nucleons $a$. Under the assumption that the collective octupole excitation does not involve spins of the individual core nucleons, we will keep only the terms that do not contain spin operators $\boldsymbol{\sigma}_{a}$ being related to the spin current of the external nucleon. Using the contact nature of the interaction (14) and taking the matrix element over the state $\psi_{b}$ of the external nucleon $b$ we can present the operator acting onto the core nucleons in the following form:

$$
\hat{W}_{a}=-\frac{G \eta_{b a}}{\sqrt{2}} \frac{1}{2 m}\left(\boldsymbol{\nabla}_{a} \cdot \psi_{b}^{\dagger}\left(\mathbf{r}_{a}\right) \boldsymbol{\sigma}_{b} \psi_{b}\left(\mathbf{r}_{a}\right)\right) .
$$

The operator $\hat{W}_{a}$ contains the octupole component proportional to the operator $\hat{O}_{\text {intr }}=$ $r^{3} Y_{30}$ and responsible for the collective excitation. To extract this component, we project out the octupole operator in the intrinsic frame,

$$
\hat{W}_{a}=C_{a} \hat{O}_{\mathrm{intr}}+\ldots,
$$

where the amplitude of this component, the contribution of a core nucleon $a$ to the octupole mode, is

$$
C_{a}=\frac{\left\langle\hat{O}_{\text {intr }} \mid \hat{W}_{a}\right\rangle}{\left\langle\hat{O}_{\text {intr }} \mid \hat{O}_{\text {intr }}\right\rangle_{R}} .
$$

Here we omitted the electric charge $e$ from the definition of the octupole operator $\hat{O}_{\text {intr }}$ since we assume that it acts both on protons and neutrons (an isoscalar octupole mode). In the collective transition to the excited octupole state the matrix element of the octupole operator $\hat{O}_{\text {intr }}$ is enhanced. Therefore, we assume that we can neglect all terms in the expansion (16) except for the one written down explicitly.

For calculating the projection constant $C$ we assume that the amplitude of the octupole vibrations is small compared to the nuclear radius so that the operator $\hat{O}_{\text {intr }}=r^{3} Y_{30}$ is acting effectively only within the nucleus, $\hat{O}_{\text {intr }}=0$ for $r>R+\delta$ where $\delta \ll R$ is some small distance. This allows us to introduce the normalized "octupole" state const $\cdot r^{3} Y_{30}$ and consistently define the projection procedure. In this case

$$
\left\langle\hat{O}_{\text {intr }} \mid \hat{O}_{\text {intr }}\right\rangle_{R}=\int_{0}^{R} r^{6} Y_{30}^{2} d^{3} r=R^{9} / 9,
$$

and 


$$
\left\langle\hat{O}_{\text {intr }} \mid \hat{W}_{a}\right\rangle=\frac{G \eta_{b a}}{\sqrt{2}} \frac{1}{2 m} \int \Psi_{b}^{+}(\mathbf{r}) \boldsymbol{\sigma} \Psi_{b}(\mathbf{r}) \cdot\left(\boldsymbol{\nabla} r^{3} Y_{30}\right) d^{3} r=\frac{9 \sqrt{7}}{40 \sqrt{2 \pi}} \frac{G \eta_{b a}}{m} R^{2} F
$$

Here we introduced the expectation value over the orbital of the external nucleon,

$$
F=\frac{\left\langle r^{2}\left(5 n_{z}^{2} \sigma_{z}-\sigma_{z}+2 n_{z}(\boldsymbol{\sigma} \cdot \mathbf{n})\right)\right\rangle}{(3 / 5) R^{2}}
$$

where $\mathbf{n}=\mathbf{r} / r$ is the unit vector, $n_{z}=\cos \theta$. Note that $\left\langle r^{2}\right\rangle \approx 3 R^{2} / 5$, therefore $F \sim 1$. In the case of ${ }^{223,225} \mathrm{Ra}$, assuming that the unpaired neutron is in the (asymptotic) Nilsson orbital $1 / 2[631]$ with $\sigma_{z}=+1$ or -1 , we would get $|F| \approx 0.3$. For a pure $l s$-state $|l m \pm\rangle$ with $m_{l}=m, s_{z}= \pm(1 / 2)$, this factor is given by

$$
\langle l m \pm|F| l m \pm\rangle= \pm 2 \frac{l(l+1)-3 m^{2}}{(2 l+3)(2 l-1)} \frac{\left\langle r^{2}\right\rangle}{(3 / 5) R^{2}} .
$$

Here the angular factor in front of $\left\langle r^{2}\right\rangle /\left(3 R^{2} / 5\right)$ changes for even $l$ from $4 / 7$ to $1 / 2$ if $m=0$ and from $-2 / 7$ to $-1 / 2$ if $m=1$. For the realistic Nilsson wave functions with the quadrupole deformation around 0.15 or 0.2 , the spin-orbital coupling is important, and the wave function of the valence neutron is a superposition of several spherical orbitals $|l, m \mp 1 / 2, \pm 1 / 2\rangle$. For example, the orbital $1 / 2[631]$ has 7 main components $\left|N l m s_{z}\right\rangle$ of comparable magnitude, where $N$ is the major oscillator quantum number,

$$
|1 / 2[631]\rangle \approx \sum_{l=0,2,4,6} a_{l}|6 l 01 / 2\rangle+\sum_{l=2,4,6} b_{l}|6 l 1-1 / 2\rangle .
$$

Although the last item in the numerator of the expression (20) contains the matrix elements with $\Delta s_{z}= \pm 1$ and is sensitive to the interference of the $\sigma_{z}= \pm$ components that might be constructive or destructive, the quantity $|F|$ is typically between 0.1 and 0.6 . For the specific values of the parameters $a_{l}$ and $b_{l}$ corresponding to the Nillson wave function 1/2[631] at $\beta_{2}=0.21$, we come to $F=-0.32$, very close to the asymptotic estimate.

Combining the equations above we obtain the effective "octupole" $\mathcal{P}, \mathcal{T}$-odd operator

$$
\hat{W}_{\text {oct }}=\frac{\left\langle\hat{O}_{\text {intr }} \mid \hat{W}_{a}\right\rangle}{\left\langle\hat{O}_{\text {intr }} \mid \hat{O}_{\text {intr }}\right\rangle_{R}} \hat{O}_{\text {intr }}=\frac{81}{40} \sqrt{\frac{7}{2 \pi}} \frac{G \eta_{b a} F}{m R^{7}} \hat{O}_{\text {intr }} .
$$

Using Eq. (13) for the proton induced transition and summing over proton and neutron contributions to the octupole excitation $\mid$ oct $\rangle$, we obtain the expression for the weak matrix element in the body-fixed frame,

$$
\langle\text { g.s. }|W| \text { oct }\rangle=\frac{81}{40} \sqrt{\frac{7}{2 \pi}} \frac{G \eta_{b} F}{m R^{7}} A R^{3} \frac{3}{4 \pi} \beta_{3}^{\text {rms }},
$$

where $\eta_{b}=\frac{Z}{A} \eta_{b p}+\frac{N}{A} \eta_{b n}, b=n$ for the external neutron or $b=p$ for the external proton.

The transition from the body-fixed frame to the laboratory frame for the vector Schiff moment gives, as in Eq. (4), an extra factor $K M / I(I+1)$ that, for the ground nuclear state, $K=M=I$, is equal to $I /(I+1)$. Collecting all factors we obtain the Schiff moment due to the octupole collective vibrations: 


$$
\begin{gathered}
\mathbf{S}=\frac{2 I}{I+1} \frac{\langle\text { g.s. }|W| \text { oct }\rangle\langle\text { oct }|\mathbf{S}| \text { g.s. }\rangle}{\Delta E}, \\
S=0.025 F \frac{I}{I+1} e \beta_{2}\left(\beta_{3}^{\text {rms }}\right)^{2} Z A^{2 / 3} \frac{\eta_{b} G}{m r_{0} \Delta E},
\end{gathered}
$$

where $\Delta E=E_{\text {g.s. }}-E_{\text {oct }}$, or, in appropriate numerical units,

$$
S=\frac{Z}{94}\left(\frac{A}{230}\right)^{2 / 3} \frac{\beta_{2}}{0.2}\left(\frac{\beta_{3}^{\mathrm{rms}}}{0.1}\right)^{2} \frac{(5 / 3)\left\langle r^{2}\right\rangle}{R^{2}} \frac{50 \mathrm{keV}}{\Delta E} \frac{I}{I+1} F\left(5650 \times 10^{-8}\right) e \eta \mathrm{fm}^{3} .
$$

The ratio of this dynamical (vibrational) octupole contribution $S_{\text {soft }}$ to the static octupole contribution $S_{\text {stat }}$ in the deformed case (10) is equal to

$$
\frac{S_{\text {soft }}}{S_{\text {stat }}}=2.5 F \frac{\left(\beta_{3}^{\text {rms }}\right)^{2}}{\left(\beta_{3}^{\text {stat }}\right)^{2}} \approx \frac{\left(\beta_{3}^{\text {rms }}\right)^{2}}{\left(\beta_{3}^{\text {stat }}\right)^{2}}
$$

This means that the dynamical deformation due to low-frequency octupole vibrations produces nearly the same effect as the static octupole deformation.

When doing numerical estimates we may assume $\beta_{3}^{\text {rms }}=\beta_{3}^{\text {stat }}=0.1$ [9, 10,11]. This gives numerical values (in the units of $10^{-8} \eta e \cdot \mathrm{fm}^{3}$ ) for the Schiff moments of ${ }^{223} \mathrm{Ra}$ and ${ }^{225} \mathrm{Ra}, S=700$ and 400, correspondingly; $S=1000$ [22 for ${ }^{223} \mathrm{Rn}$; and in the interval $100-1000$ for ${ }^{223} \mathrm{Fr},{ }^{225} \mathrm{Ac}$ and other nuclei with the low octupole mode, $\Delta E<100 \mathrm{keV}$, i.e. close to the values [9, 10] obtained in the numerical calculations for the static octupole deformation. However, our value for ${ }^{239} \mathrm{Pu}$, where $\Delta E=470 \mathrm{keV}$, is $S \sim 80$, or even smaller if we take into account that in the ground state of a harmonic oscillator the vibrational amplitude is proportional to $\omega^{-1 / 2}$ so that $\left\langle\beta^{2}\right\rangle \propto 1 / \omega$ as it is approximately the case for both quadrupole and octupole low-lying vibrations in nuclei [23,24]. This result is much smaller than the estimates $S(\mathrm{Pu})=300 \cdot S(\mathrm{Hg}) \approx 420$ presented in Ref. [11.

The work [11 also claims that the contribution of the octupole vibration to the ${ }^{199} \mathrm{Hg}$ Schiff moment is about half of its shell model value. Strictly speaking, our formula is not applicable for this case since ${ }^{199} \mathrm{Hg}$, probably, has no quadrupole deformation. However, for an estimate we can take $\beta_{2}=0.1$ assuming that a similar contribution may appear due to an interaction with soft quadrupole vibrations. Now we can make a comparison between ${ }^{225} \mathrm{Ra}$ and ${ }^{199} \mathrm{Hg}$ which both have $I=1 / 2$. The octupole transition energy in ${ }^{199} \mathrm{Hg}$ is $3000 \mathrm{keV}$ [11]. Therefore, in the case of ${ }^{199} \mathrm{Hg}$ we lose one-two orders of magnitude due to the octupole deformation [if $\beta_{3}^{2} \propto(\Delta E)^{-1}$ ] and one-two orders of magnitude due to the energy denominator that is sixty times greater than in ${ }^{223,225} \mathrm{Ra}$. This gives the octupole contribution to the ${ }^{199} \mathrm{Hg}$ Schiff moment about $400 \cdot 0.001 \sim 0.4$. The shell-model contribution is -1.4 . This estimate shows that the octupole contribution should not produce any dramatic changes in the ${ }^{199} \mathrm{Hg}$ Schiff moment (in fact, this conclusion agrees with that of Ref. [11]).

\section{B. Particle excitation}

In the previous consideration the orbital of the valence nucleon in the mean field of a normal quadrupole deformation was fixed. In order to estimate the contribution of the 
abovementioned second mechanism related to the mixing of single-particle orbitals by the octupole mode, we assume that the octupole component of the strong nuclear Hamiltonian can be estimated in terms of octupole-octupole forces [20] as $\beta_{3} V_{3}$, where $\beta_{3}$ acts on the core variables exciting octupole vibrations while $V_{3}=f(r) Y_{30}$ is the perturbation acting on the valence nucleons. For the small amplitude $\beta_{3}, f(r)$ is proportional to the derivative of the mean nuclear potential. As pointed out in Ref. [11, this interaction mixes the ground state $|\lambda ; 0\rangle$ of an odd- $A$ nucleus (a valence particle on a single-particle orbital $\lambda$ and the even-even core in the ground state) with the excited states $|\nu ; 1\rangle$ that contain a particle in an orbital $\nu$ of opposite parity and a phonon of the octupole mode. To the first order in this mixing, the new ground state is

$$
|\tilde{0}\rangle=|\lambda ; 0\rangle+\sum_{\nu} c_{\nu}|\nu, 1\rangle
$$

The admixture coefficients are given by

$$
c_{\nu}=\frac{\left\langle 1\left|\beta_{3}\right| 0\right\rangle\left(V_{3}\right)_{\nu \lambda}}{\epsilon_{\lambda}-\epsilon_{\nu}-\hbar \omega}
$$

where $\epsilon_{\lambda}$ and $\epsilon_{\nu}$ are single-particle energies whereas $\hbar \omega$ is energy of the octupole core excitation. Similarly, the unperturbed one-phonon state $|\lambda ; 1\rangle$ also acquires new components being converted into

$$
|\tilde{1}\rangle=|\lambda ; 1\rangle+\sum_{\nu} c_{\nu}^{\prime}|\nu ; 0\rangle
$$

where

$$
c_{\nu}^{\prime}=\frac{\left\langle 0\left|\beta_{3}\right| 1\right\rangle\left(V_{3}\right)_{\nu \lambda}}{\epsilon_{\lambda}-\epsilon_{\nu}+\hbar \omega}
$$

and we omit the two-phonon admixtures irrelevant for our purpose.

The states $|\tilde{0}\rangle$ and $|\tilde{1}\rangle$ can be mixed by the effective $\mathcal{P}, \mathcal{T}$-odd potential $W$ averaged, in distinction to the previous case of Eq. (15), over the core nucleons. For a conventional definition of angular wave functions, the matrix elements of $\beta_{3}, V_{3}$ and $W$ are real, and the mixing matrix element is

$$
\langle\tilde{0}|W| \tilde{1}\rangle=\left\langle 1\left|\beta_{3}\right| 0\right\rangle \sum_{\nu} \frac{2\left(\epsilon_{\lambda}-\epsilon_{\nu}\right)}{\left(\epsilon_{\lambda}-\epsilon_{\nu}\right)^{2}+(\hbar \omega)^{2}} W_{\lambda \nu}\left(V_{3}\right)_{\nu \lambda} \equiv\left\langle 1\left|\beta_{3}\right| 0\right\rangle\left\langle\tilde{0}\left|W_{0}\right| \tilde{0}\right\rangle .
$$

where the single-particle matrix elements can be renormalized by the pairing correlations. In the limit of a soft octupole mode, when $(\hbar \omega)^{2}$ is small compared to the typical single-particle energy intervals $\left(\epsilon_{\lambda}-\epsilon_{\nu}\right)^{2}$ between the orbitals of opposite parity, the latter expression coincides with the result for the static octupole deformation found in Ref. [10]. This leads to a conclusion that the second mechanism in the presence of the soft octupole mode provides the Schiff moment

$$
S_{2} \approx \frac{2 I}{I+1} \frac{\left|\left\langle 1\left|\beta_{3}\right| 0\right\rangle\right|^{2}}{\Delta E} \frac{9}{20 \pi \sqrt{35}} e Z R^{3} \beta_{2}\left\langle\tilde{0}\left|W_{0}\right| \tilde{0}\right\rangle
$$


that can be described by Eq. (10), derived for the static octupole contribution, with the replacement of $\left(\beta_{3}^{\text {stat }}\right)^{2}$ by $\left(\beta_{3}^{\text {rms }}\right)^{2}=\left|\left\langle 1\left|\beta_{3}\right| 0\right\rangle\right|^{2}$. It is easy to explain this result. For an adiabatically slow vibrational motion, we can calculate the weak and Schiff moment matrix elements at a fixed value of $\beta_{3}$ and then average over $\beta_{3}$. This conclusion holds for the first mechanism as well. Indeed, keeping the linear in $\beta_{3}$ terms, we can present the effective $\mathcal{P}, \mathcal{T}$-odd potential $W$ averaged over the core nucleons as $W=W_{0}+\beta_{3} W_{3}$. This leads to the following contribution of the octupole weak field

$$
S_{2} \approx \frac{2 I}{I+1} \frac{\left|\left\langle 1\left|\beta_{3}\right| 0\right\rangle\right|^{2}}{\Delta E} \frac{9}{20 \pi \sqrt{35}} e Z R^{3} \beta_{2}\left\langle 0\left|W_{3}\right| 0\right\rangle
$$

Here the expectation value is taken over the ground state $\lambda$ of the valence nucleon $\left(\left\langle 0\left|W_{3}\right| 0\right\rangle=\left\langle\lambda\left|W_{3}\right| \lambda\right\rangle\right)$. Again, the replacement of $\left(\beta_{3}^{\text {rms }}\right)^{2}=\left|\left\langle 1\left|\beta_{3}\right| 0\right\rangle\right|^{2}$ by $\left(\beta_{3}^{\text {stat }}\right)^{2}$ reduces the dynamical problem to the static one. The final result for the first mechanism contribution was given in eq. (26).

\section{FROM NUCLEAR SCHIFF MOMENT TO ATOMIC EDM}

Finally, we should calculate the atomic electric dipole moments induced by the nuclear Schiff moments.

The atomic EDM is generated by the $\mathcal{P}, \mathcal{T}$-odd part of the nuclear electrostatic potential $\varphi(\mathbf{r})$. The potential produced by the point-like Schiff moment is usually presented in the form [6] proportional to the gradient of the delta-function at the origin,

$$
\varphi(\mathbf{r})=4 \pi \mathbf{S} \cdot \boldsymbol{\nabla} \delta(\mathbf{r}) .
$$

The natural generalization of the Schiff moment potential for a finite-size nucleus is [12]

$$
\varphi(\mathbf{r})=-3(\mathbf{S} \cdot \mathbf{r}) \frac{n(r)}{B},
$$

where $B=\int n(r) r^{4} d r \approx R^{5} / 5, R$ is the nuclear radius, and $n(r)$ is a smooth function which is 1 for $r<R-\delta$ and 0 for $r>R+\delta$ while $\delta \ll R$ is some small distance; $n(r)$ can be taken as proportional to the nuclear density. The potential (37) corresponds to a constant electric field $\mathcal{E}$ inside the nucleus that is directed along the nuclear spin, $\mathcal{E} \propto \mathbf{I}$. The interaction $-e \varphi$ mixes electron orbitals of opposite parity and produces EDMs in atoms.

In Ref. [14] we have performed atomic calculations of the coefficients $k$ that define the atomic EDM $d$ in terms of the Schiff moments,

$$
d=k \cdot 10^{-17} \cdot\left(\frac{S}{e \cdot \mathrm{fm}^{3}}\right) e \cdot \mathrm{cm} .
$$

The factors $k$ rapidly grow with the nuclear charge that leads to an additional enhancement in Ra, $k=-8.5$, and $\mathrm{Rn}, k=3.3$, in comparison with the lighter electronic analogues, $\mathrm{Hg}$, $k=-2.8$, and $\mathrm{Xe}, k=0.38$.

We have estimated above the individual contributions of two possible mechanisms for the enhancement of original $\mathcal{P}, \mathcal{T}$ - odd interactions $W$ by nuclear soft octupole degrees of 
freedom. The virtual core excitation and the mixing of single-particle states work in fact together and lead to the resulting enhancements of the same order of magnitude. In principle they may involve different parts of the interaction $W$. The detailed calculation of the total result is subject to many uncertainties both on the fundamental and nuclear structure levels; this should be studied separately. However, there is no physical reason for expecting their strong cancellation. As we have seen in the consideration of the core excitation mechanism, the exact magnitude of the resulting Schiff moment is quite sensitive to the details of the valence single-particle wave function in the presence of spin-orbit coupling, whereas the second mechanism is determined mainly by the intruder orbits of opposite parity. With no accidental cancellation, the resulting Schiff moment will be of the same order of magnitude as each of the contributions. Taking some conservative "minimal" values of the Schiff moments for the different methods of calculations (static analytical, static numerical, soft analytical) we obtain the values of the atomic electric dipole moments given in the Table.

\begin{tabular}{c|c|c} 
Nucleus & $S, 10^{-8} \eta_{n} e \cdot \mathrm{fm}^{3}$ & $d, 10^{-25} e \cdot \mathrm{cm} \cdot \eta_{n}$ \\
\hline${ }^{225} \mathrm{Ra}$ & 300 & 2500 \\
${ }^{223} \mathrm{Ra}$ & 400 & 3400 \\
${ }^{223} \mathrm{Rn}$ & 1000 & 3300
\end{tabular}

This may be compared with typical values of the induced dipole moment predicted for spherical nuclei, $d=4$ and $d=0.7$ for ${ }^{199} \mathrm{Hg}$ and ${ }^{129} \mathrm{Xe}$, respectively.

To conclude, we made analytical estimates for the nuclear Schiff moment in nuclei known as soft with respect to the octupole excitation mode. We found a strong enhancement of the average magnitude close to what was found for nuclei having static octupole deformation. There are several factors which contribute to this enhancement. Mainly, they are small energy interval between the opposite parity states and large amplitude of vibrations in the case of the soft octupole mode. Effectively, the enhancement factor can be presented as const $\times(E / \hbar \omega)^{2}$, where $E$ is the intershell distance in spherical nuclei, $\omega$ is the frequency of the octupole mode and const $\simeq 0.1$. The value of the Schiff moment is typically $10^{2} \div 10^{3}$ in units of $10^{-8} \eta e \cdot \mathrm{fm}^{3}$; it leads to the prediction of the enhancement of the atomic EDM on the level of $10^{3}$ as compared to spherical nuclei.

A related idea should be explored in the future: it is known that some nuclei are soft with respect to both quadrupole and octupole modes, see for example recent predictions for radioactive nuclei along the $N=Z$ line [25]. The light isotopes of $\mathrm{Rn}$ and $\mathrm{Ra}$ are spherical but with a soft quadrupole mode and therefore large amplitude of quadrupole vibrations. The spectra of these nuclei display long quasivibrational bands based on the ground state and on the octupole phonon, with positive and negative parity, respectively. These bands are connected via low-energy electric dipole transitions. This situation seems to be favorable for the enhancement of $\mathcal{P}, \mathcal{T}$-odd effects.

The authors are thankful to the Institute of Nuclear Theory, University of Washington, where this work was started, for hospitality and support. The discussions with A. Dieperink, O. Scholten and J. Engel are gratefully acknowledged. V.Z. acknowledges support from the NSF grant PHY0070911. V.F. acknowledges support from the Australian Research Council. He is also grateful to the Institute for Theoretical Atomic and Molecular Physics, Harvard-Smithsonian Center for Astrophysics, where part of this work has been done. 


\section{REFERENCES}

[1] E.M. Purcell and N.F. Ramsey, Phys. Rev. 78, 807 (1950).

[2] M.V. Romalis, W.C. Griffith, J.P. Jacobs, and E.N. Fortson, Phys. Rev. Lett. 86, 2505 (2001).

[3] V.V. Flambaum, I.B. Khriplovich and O.P. Sushkov, Nucl. Phys. A449, 750 (1986).

[4] L.I. Schiff, Phys. Rev. 132, 2194 (1963).

[5] P.G.H. Sandars, Phys. Rev. Lett. 19, 1396 (1967).

[6] V.V. Flambaum, I.B. Khriplovich, and O.P. Sushkov, J. Exptl. Theor. Phys. 87, 1521 (1984) [English translation JETP 60, 873 (1984)].

[7] W.C. Haxton and E.M. Henley, Phys. Rev. Lett. 51, 1937 (1983).

[8] V.V. Flambaum and V.G. Zelevinsky, Phys. Lett. B 350, 8 (1995).

[9] N. Auerbach, V.V. Flambaum, and V. Spevak, Phys. Rev. Lett. 76, 4316 (1996).

[10] V. Spevak, N. Auerbach, and V.V. Flambaum, Phys. Rev. C 56, 1357 (1997).

[11] J. Engel, J.L. Friar, and A.C. Hayes, Phys. Rev. C 61, 035502 (2000).

[12] V.V. Flambaum and J.S.M. Ginges, Phys. Rev. A 65, 032113 (2002).

[13] Note that the numerical calculation in [10] includes effects of the octupole deformation in both the strong and weak potentials - see Eqs. (30)-(31) in [10. This is important since these two contributions tend to cancel each other. A comment in [11] that the second contribution was neglected in [10] is erroneous.

[14] V.A. Dzuba, V.V. Flambaum, J.S.M. Ginges, and M.G. Kozlov. Phys. Rev. A 66, 012111 (2002).

[15] V.V. Flambaum, Phys. Rev. A 60, R2611 (1999).

[16] V.A. Dzuba, V.V. Flambaum, and J.S.M. Ginges, Phys. Rev. A 61, 062509 (2000).

[17] V.V. Flambaum. Phys. Lett. B 320 , 211 (1994).

[18] V.V. Flambaum, D.W. Murray, and S.R. Orton, Phys. Rev. C 56, 2820 (1997).

[19] G.A. Leander and Y.S. Chen, Phys. Rev. C 37, 2744 (1988).

[20] A. Bohr and B. Mottelson, Nuclear Structure (Benjamin, New York, 1975), Vol. 2.

[21] R.H. Spear, At. Data and Nucl. Data Tables 42, 55 (1989).

[22] The energy denominator for ${ }^{223} \mathrm{Rn}$ is not known experimentally and the particle-rotor model [19] does not give a unique prediction. Therefore we take the energy splitting 50 $\mathrm{keV}$, similar to ${ }^{223} \mathrm{Ra}$ and ${ }^{225} \mathrm{Ra}$. The calculated value of $37 \mathrm{keV}$ [10 is even smaller.

[23] S. Raman, C.W. Nestor, Jr., S. Kahane, and K.H. Bhatt, Phys. Rev. C 43, 556 (1991).

[24] M.P. Metlay, J.L. Johnson, J.D. Canterbury, P.D. Cottle, C.W. Nestor, Jr., S. Raman, and V.G. Zelevinsky, Phys. Rev. C 52, 1801 (1995).

[25] K. Kaneko, M. Hasegawa, and T. Mizusaki, nucl-th/0211021. 